\title{
Osteoporosis as a side effect of antineoplazic therapy
}

El Hajj Sleiman Joan ${ }^{1,2}$, Dan Jinga ${ }^{1,3}$, Cornelia Nitipir ${ }^{1,2}$, Carmen Gabriela Barbu ${ }^{1,2}$

${ }^{1}$ Carol Davila University of Medicine and Pharmacy, Bucharest, Romania,

2Elias Hospital, Bucharest,Romania,

3Universitary Hospital,Bucharest, Romania

Conflict of interest: All the authors - nothing to disclosure

Objective: The aim of this retrospective study is to evaluate the bone status in patients receiving oncological treatment, like radiation, chemotherapy, adjunctive therapies, and surgery.

Material and methods. Medical records of 102 womens (mean age $59.12 \mathrm{yrs}$ ) with breast cancer history reffered for endocrine evaluation were retrospectivelly analysed. Demographic data, bone densitometry parameters, prevalent fractures and antineoplastic treatments history were collected.

\section{Results:}

All of the patients had history of breast surgery and chemotherapy; $10 \%$ had also radiotherapy and $87 \%$ had a form of hormone suppresive treatment. According to the lowest T score, 33\% had densitometric criteria for osteoporosis and $55 \%$ had osteopenia; $13.1 \%$ of the subjects had prevalent fractures at the evaluation moment. $31 \%$ of the patients had the lowest Z score less than -1SD in the absence of early menopause. According to our data, the prevalence of decrese in BMD in serial mesurements was $40.5 \%$ in chemotherapy only patients, $62 \%$ in Al only patients and $37.5 \%$ in patients with sequential combination between SERM and AI. Only 4 patients out of 34 osteoporotic patients received treatment for their osteoporosis.
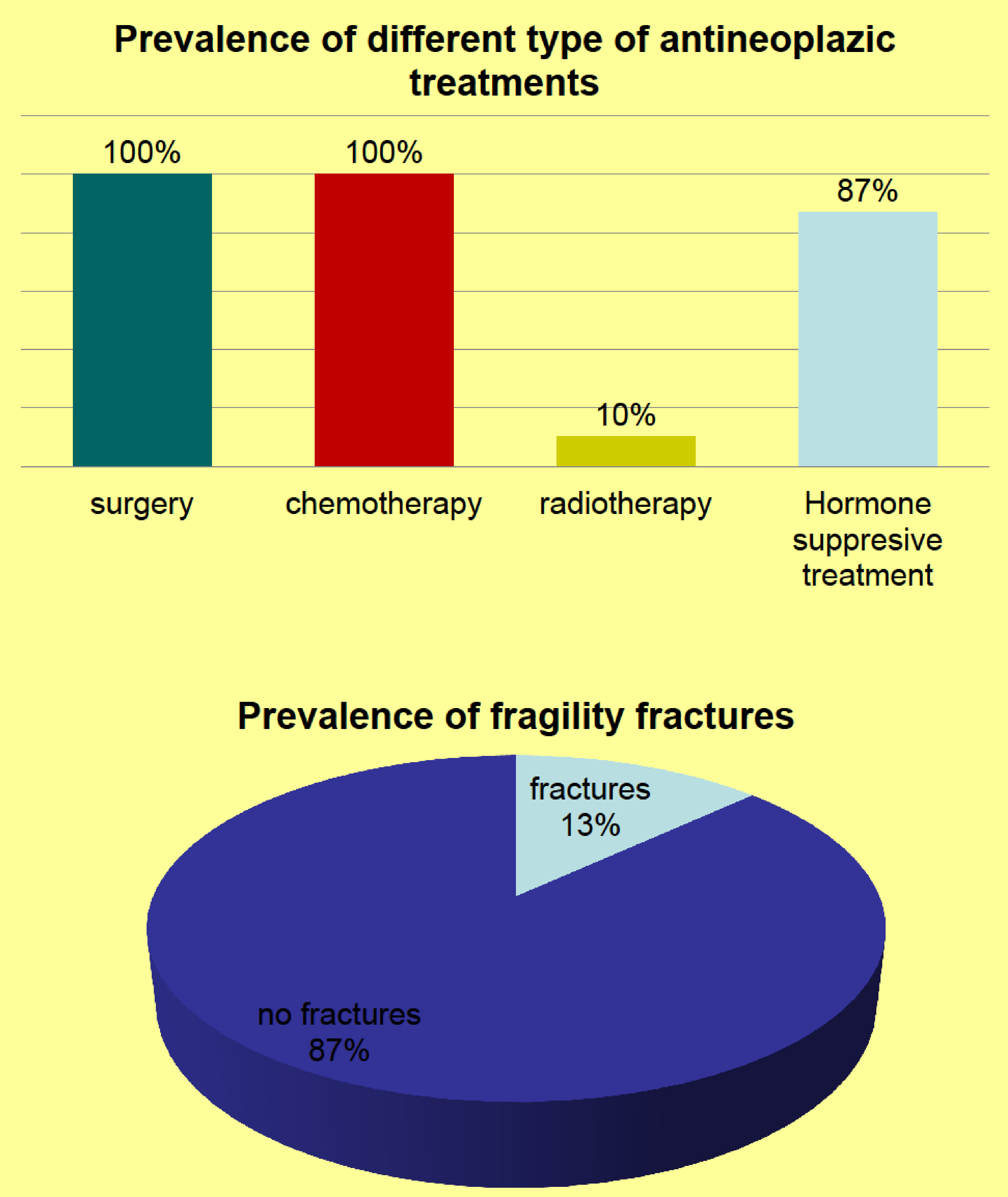

\section{Conclusion.}

Our data suggest an increased prevalence of decrease in BMD in patients related to their history of antineoplazic treatments; from all the combinations, chemotherapy alone had almost the same effect as sequential combination of SERM and $\mathrm{Al}$ and the most agresive for the bone was proved to be chemotherapy and Al treatment.

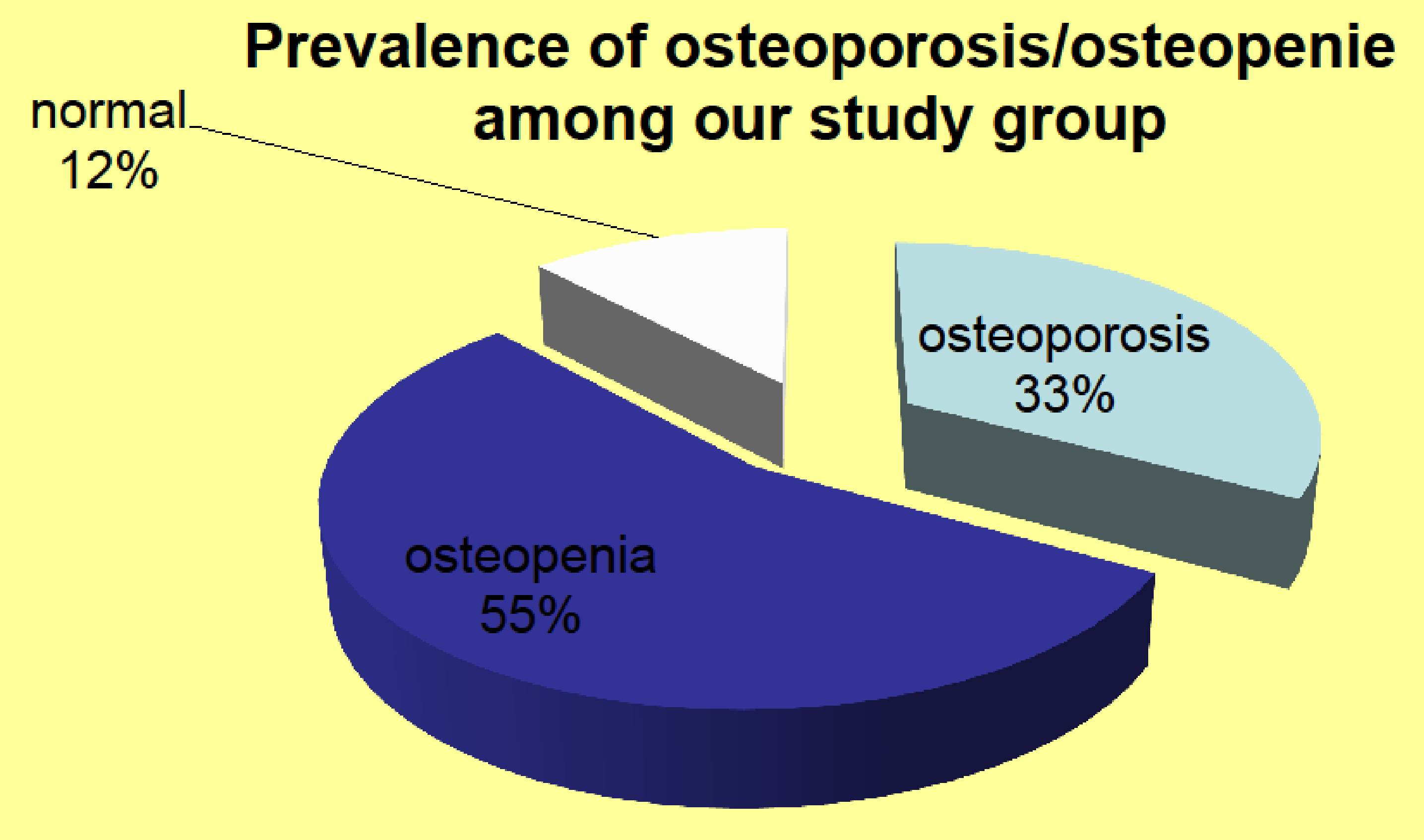

Prevalence of low $Z$ score

lowest $Z$ score less than -1SD in the absence of early menopause normal

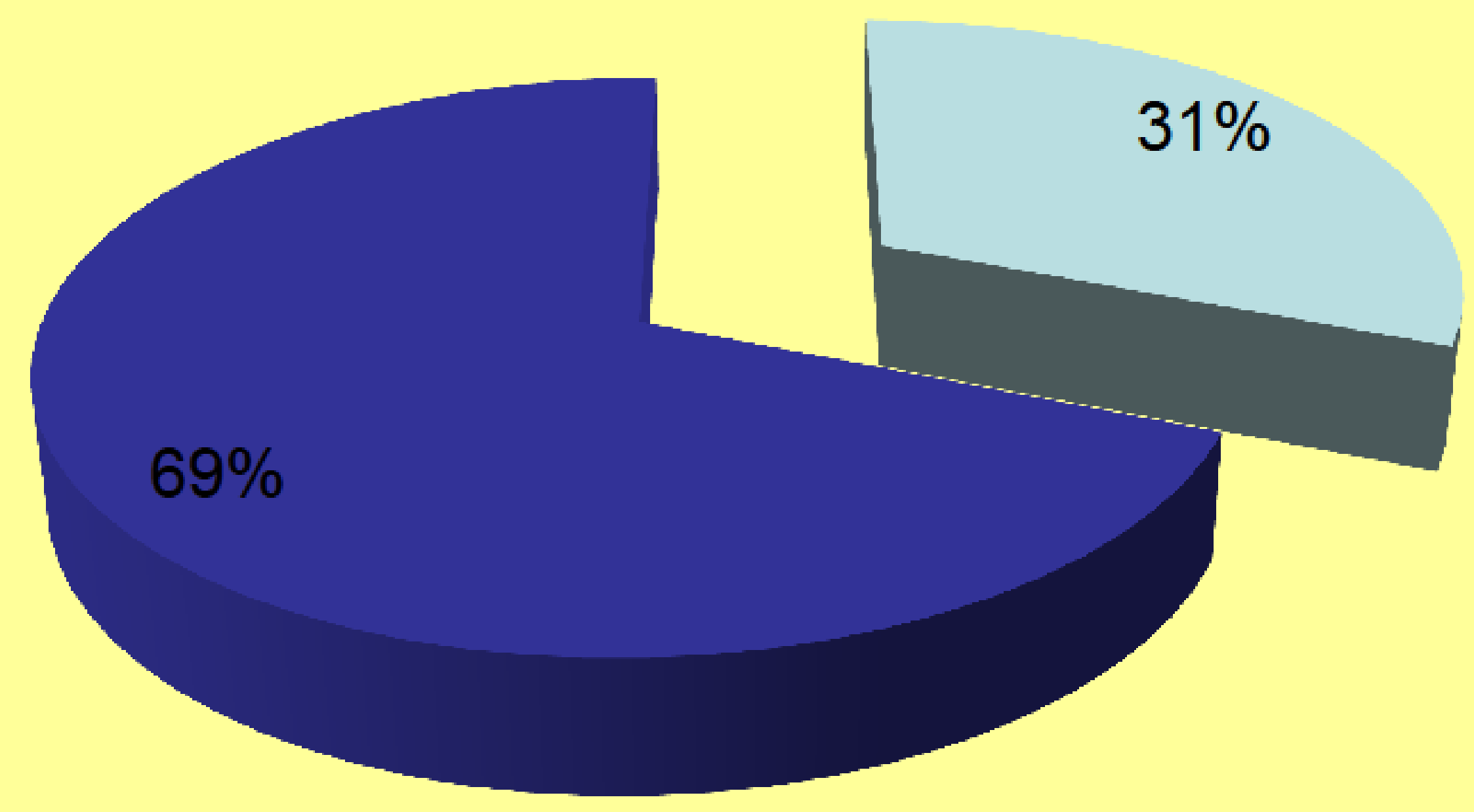

BMD evolution

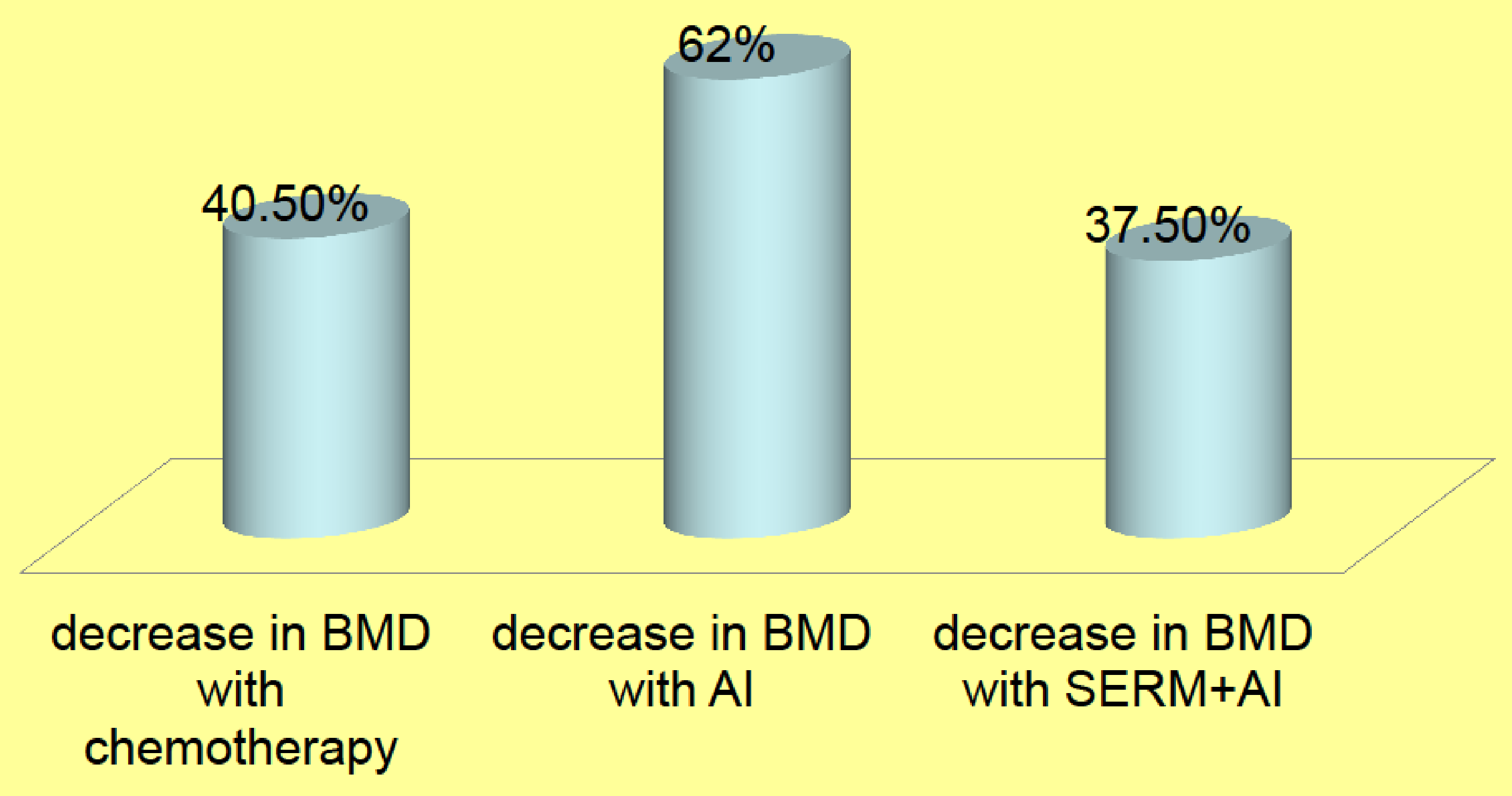

